\title{
Split-hand and split-limb phenomena in amyotrophic lateral sclerosis: pathophysiology, electrophysiology and clinical manifestations
}

\author{
Philippe Corcia (D) , ${ }^{1}$ Peter Bede (D) , 2,3 Pierre-François Pradat, ${ }^{4,5}$ Philippe Couratier, ${ }^{6}$ \\ Steve Vucic (10) 7,8 Mamede de Carvalho ${ }^{9,10}$
}

${ }^{1}$ Centre Constitutif de Référence SLA, CHU Bretonneau, Tours, France

${ }^{2}$ Computational Neuroimaging Group, Trinity College Dublin, Ireland

${ }^{3}$ Pitié-Salpêtrière University Hospital, Sorbonne University, Paris, France

${ }^{4}$ Neurology, Hopital PitieSalpetriere, Paris, France

${ }^{5} \mathrm{LIB}$, Université Pierre et Marie Curie Faculté de Médecine

Paris, Île-de-France, France ${ }^{6}$ Centre Constitutif de Référence SLA, CHU de Limoges, Limoges, France

${ }^{7}$ Westmead Clinical School, Westmead Hospital, University of Sydney, Sydney, New South Wales, Australia

${ }^{8}$ Sydney Medical School, The University of Sydney, Sydney, New South Wales, Australia

${ }^{9}$ Instituto de Fisiologia, Instituto de Medicina Molecular,

Faculdade de Medicina, Universidade de Lisbon, Lisbon, Portugal

${ }^{10}$ Department of Neurosciences and Mental Health, Hospital de Santa Maria, Lisboa, Portugal

\section{Correspondence to}

Professor Philippe Corcia, ALS Center, Université de Tours Faculté de Médecine, Tours, Centre-Val de Loire, France; corcia@med.univ-tours.fr

Received 1 May 2021 Accepted 5 July 2021 Published Online First 20 July 2021

Check for updates

(C) Author(s) (or their employer(s)) 2021. No commercial re-use. See rights and permissions. Published by BMJ.

To cite: Corcia $\mathrm{P}$, Bede $\mathrm{P}$ Pradat P-F, et al. J Neurol Neurosurg Psychiatry 2021:92:1126-1130.

\section{ABSTRACT}

Amyotrophic lateral sclerosis (ALS) is a progressive neurodegenerative disorder affecting the upper and lower motor neurons. A key clinical feature of ALS is the absence of accurate, early-stage diagnostic indicators. 'Split-hand syndrome' was first described in ALS at the end of the last century and a considerable body of literature suggests that the split-hand phenomenon may be an important clinical feature of ALS. Considering the published investigations, it is conceivable that the 'splithand syndrome' results from the associated upper and lower motor neuron degeneration, whose interaction remains to be fully clarified. Additionally, other split syndromes have been described in ALS involving upper or lower limbs, with a nuanced description of clinical and neurophysiological manifestations that may further aid ALS diagnosis. In this review, we endeavour to systematically present the spectrum of the 'split syndromes' in ALS from a clinical and neurophysiology perspective and discuss their diagnostic and pathogenic utility.

\section{INTRODUCTION}

Amyotrophic lateral sclerosis (ALS) is a relentlessly progressive neurodegenerative disorder characterised by coexisting upper (UMN) and lower (LMN) motor neuron degeneration with a median survival of 36 months. ${ }^{1}$ Although site of onset and rate of progression are variable, disease onset is often focal, typically involving the distal segments of upper or lower limbs. A dissociated pattern of muscle atrophy is classically observed in the hands, more severe in the lateral hand group of muscles, predominantly affecting the thenar eminence (abductor pollicis brevis (APB)) and first dorsal interosseous muscle (FDI), with relative preservation of the medial hand, particularly the abductor digit minimi (ADM) (figure 1, table 1). The pattern of dysfunction does not respect nerve trunk or root territories since both the FDI and ADM muscles are innervated by the ulnar nerve arising from the same nerve roots (C8-T1). This intriguing semiology was referred to as 'split-hand' by Wilbourn more than 30 years ago. ${ }^{2}$

The 'split-hand' phenomenon is observed in around $70 \%$ of patients with ALS at time of diagnosis and is evident in almost all cases at some stage of the disease. ${ }^{3}$ While the split-hand phenomenon is more commonly observed in spinal-onset ALS, it is also observed in bulbar onset ALS as well as patients in early stages of the disease as defined by functional deficits, making it a potentially important diagnostic indicator. ${ }^{45}$

Split-hand ratio and index

Neurophysiology studies helped to define two main ratios and one index, which may confirm and quantify the severity of split-hand syndrome. The ratios are derived from the compound muscle amplitude potential (CMAP) amplitude of the relevant hand muscles, using different formulas (APB/ $\mathrm{ADM}$ or FDI/ADM). Ratio values of less than 0.6 and 0.9 , respectively, or an $\mathrm{ADM} / \mathrm{APB}$ value higher than 1.7 were considered abnormal. Clinical studies suggested very good diagnostic utility of this simple neurophysiological test, irrespective of disease onset site, with the ADM/APB CMAP amplitude ratio $>1.7$ or absent APB CMAP response yielding a 51\% sensitivity and $91 \%$ specificity when compared with ALS mimic disorders. ${ }^{6}$ Of relevance, an extremely high ADM/APB CMAP amplitude ratio $(\geq 4.5)$ or an absent APB CMAP was exclusively observed in patients with ALS. ${ }^{5}$ In a smaller study, the ADM/ APB CMAP amplitude ratio of $>1.7$ was reported to exhibit moderate sensitivity (60\%) and high specificity (90.2\%) for ALS, thereby further supporting its use as a diagnostic aid in ALS. ${ }^{6}$

The split-hand index (SI) is derived by multiplying the APB and FDI CMAP amplitudes and dividing the product by the ADM CMAP amplitude according to the following formula: $\left(\mathrm{SI}_{\mathrm{CMAP}}=\mathrm{APB}\right.$ ${ }_{\text {CMAP }} \times \mathrm{FDI}_{\text {CMAP }}\left(\mathrm{ADM}_{\mathrm{CMAP}}\right)$. The diagnostic utility of the SI was confirmed in a large study, with a cutoff value $<5.2$ exhibiting a sensitivity of $74 \%$ and specificity of $80 \%$ when compared with mimicking disorders. ${ }^{3}$ This was confirmed in a subsequent study, with the SI being more sensitive and specific for ALS when compared with neuromuscular mimicking disorders. ${ }^{6} 7$ Importantly, a reduced SI was evident in $64 \%$ of patients with ALS not meeting the Awaji criteria, ${ }^{3}$ thereby suggesting a diagnostic utility in early stages of ALS. ${ }^{3}$

A potential limitation of the neurophysiological indices pertains to their reliance on absence of median and ulnar entrapment neuropathies as well as marked intrinsic muscle atrophy. Consequently, these neurophysiological indices should be applied in a clinical context and not used in isolation.

An adapted version of the SI was developed by evaluating $\mathrm{F}$-wave persistence from the target muscles and based on observations of reduced 


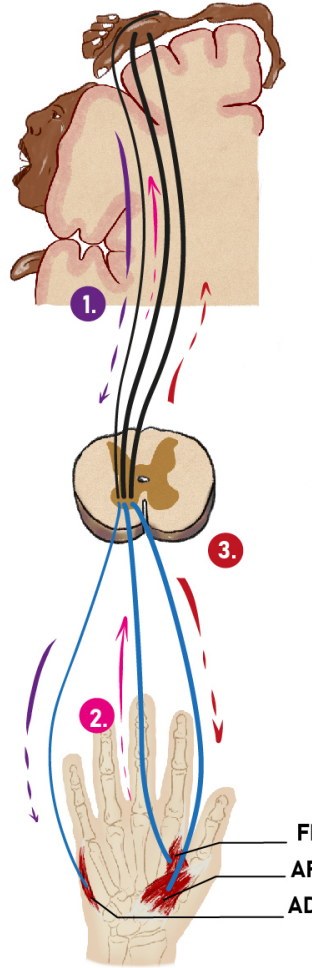

A

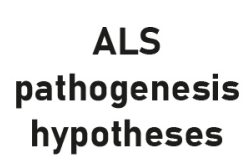

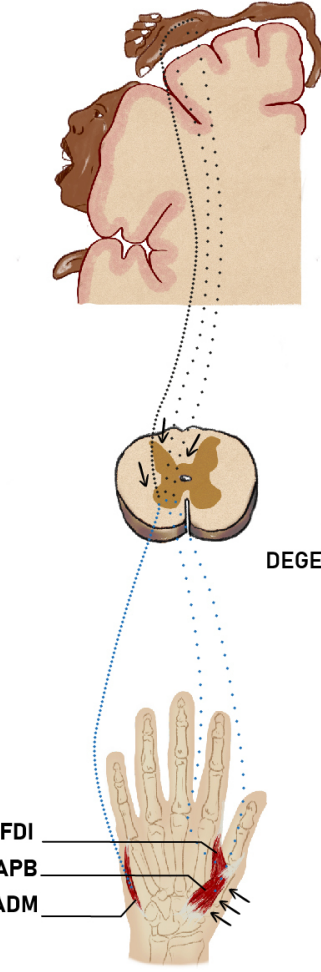

B

CORTICAL

HYPER

EXCITABILITY

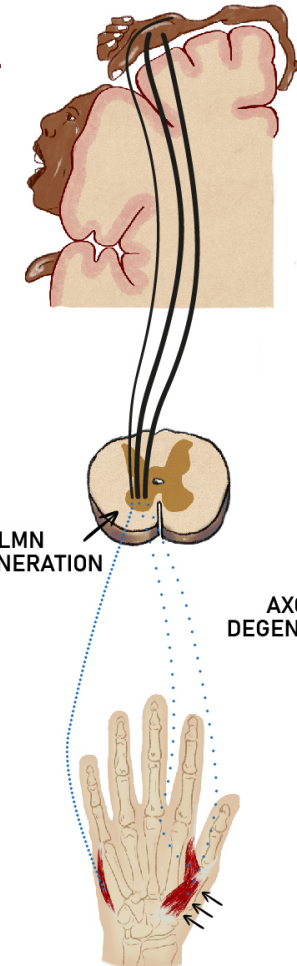

C

ABNORMAL

SPINAL

CIRCUITRY

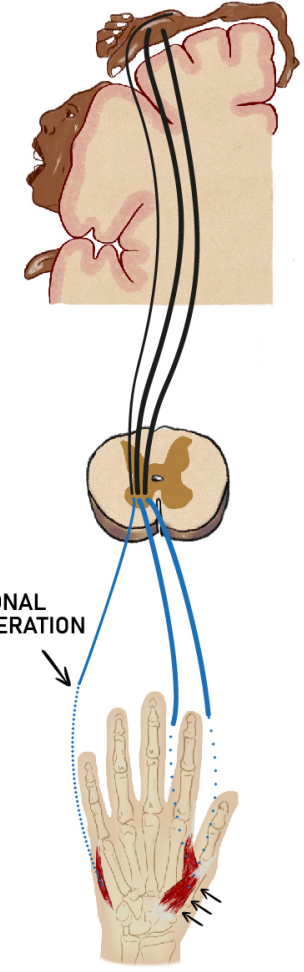

D

AXONAL MEMBRANE CHANNEL DYSFUNCTION

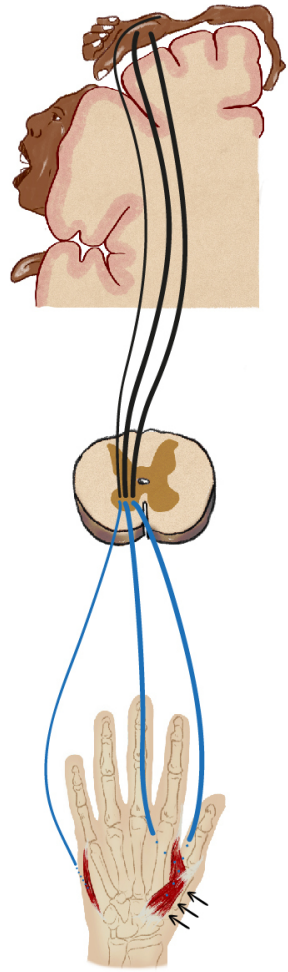

E

END PLATE

PHYSIOLOGICAL FEATURES

\section{Split hand pathogenesis hypotheses}

Figure 1 Pathophysiological hypotheses of the split-hand syndrome. (1) Dying forward: hypothesis proposes that corticomotoneurons which connect monosynaptically with anterior horn cells, mediate neurodegeneration via anterograde glutamatergic excitotoxic process. (2) Dying back hypothesis proposes that ALS begins in muscles or neuromuscular junction. (3) Independent degeneration hypothesis proposes that upper and lower motor neuron degeneration occurs independently. A: ALS pathogenesis hypotheses. Dying forward: hypothesis proposes that corticomotoneurons, which connect monosynaptically with anterior horn cells, mediate neurodegeneration via anterograde glutamatergic excitotoxic process. Dying back hypothesis proposes that ALS begins in muscles or neuromuscular junction. Independent degeneration hypothesis proposes that upper and lower motor neuron degeneration occurs independently. B-E: split-hand pathogenesis hypotheses. B: cortical dysfunction; C: abnormal spinal circuitry; D: axonal membrane channel dysfunction; E: endplate physiological features. ALS, amyotrophic lateral sclerosis.

F-wave persistence when recording over the FDI muscle compared with ADM. ${ }^{78}$ While the sensitivity of the F-wave method (85.7\%) was higher when compared with CMAP method $(75.8 \%)$, the requirement to deliver 100 supramaximal stimuli for each tested muscle limits the clinical utility. ${ }^{7}$
More recently, the motor unit number index (MUNIX) was also explored as a method for determining the split-hand phenomenon. MUNIX is a non-invasive, reliable and standardised technique for determining motor unit number. ${ }^{9}$ The MUNIX based SI was determined by multiplying the MUNIX values from the

\begin{tabular}{|c|c|c|c|c|c|}
\hline & Value & Sensitivity (\%) & Specificity (\%) & Control population & Ref. \\
\hline & \multicolumn{5}{|l|}{ Based on CMAP } \\
\hline APB/ADM & $<0.6$ & 40 & 91.0 & DC & 4 \\
\hline ADM/APB & $>1.7$ & 51.0 & 99 & HC & 5 \\
\hline ADM/APB & $>1.7$ & 58.1 & 95 & HC & 6 \\
\hline FDI/ADM & $<0.9$ & 34 & 63 & $D C$ & 4 \\
\hline \multirow[t]{2}{*}{ APB/ADM FDI/ADM } & $<0.6$ and $<0.9$ & 20 & 94 & $D C$ & 4 \\
\hline & \multicolumn{5}{|c|}{ Based on split index } \\
\hline SI CMAP & $<5.2$ & 74 & 80 & $D C$ & 3 \\
\hline SI CMAP & $<6.4$ & 62 & 80 & DC & 14 \\
\hline SI FP & $\leq 80.7$ & 81.2 & 97.0 & $\mathrm{HC}$ & 7 \\
\hline SI MUNIX & $\leq 9.4$ & 85.0 & 79.5 & $\mathrm{HC}$ & 10 \\
\hline
\end{tabular}

ADI, abductor digit minimi; APB, abductor pollicis brevis; CMAP, compound muscle amplitude potential; DC, disease controls; FDI, first dorsal interosseous muscle; HC, healthy controls; MUNIX, motor unit number index; SI FP, split index from $\mathrm{F}$-wave persistence. 


\section{Box 1 Reverse split-hand syndrome}

- Predominant atrophy and weakness of the medial hand segment.

- Strongly suggestive of Hirayama disease.

- ADM/APB ratio $<0.6$, sensitivity $52.9 \%$; specificity $97.4 \%$.

- ADM/APB ratio $<0.86$, sensitivity $80.4 \%$; specificity $86.3 \%$.

- Observed in cervical spondylotic amyotrophy.

- Rare $(<4 \%)$ in ALS.

ADI, abductor digit minimi; ALS, amyotrophic lateral sclerosis; APB, abductor pollicis brevis.

APB and FDI muscles and dividing the product by the MUNIX value form the ADM, analogous the original SI method. When compared with healthy controls, the MUNIX method exhibited high sensitivity $(95 \%)$ and specificity $(84.6 \%) .{ }^{10}$ Moreover, the MUNIX method detected the split-hand pattern in early stages of ALS in the setting of normal CMAP amplitudes. While the method is promising, further confirmation of its diagnostic utility is required in larger ALS cohorts with comparisons to neuromuscular mimicking disorders rather than healthy controls.

\section{OTHER SPLIT PHENOTYPES IN THE UPPER LIMBS}

Although split-hand syndrome is primarily associated with ALS, split-hand has been observed in other conditions, such as spinal muscular atrophy (SMA), spinocerebellar ataxia type-3, Kennedy's disease (SBMA) or postpolio syndrome. ${ }^{11-14}$ A recent study suggested that the split-hand syndrome may be a feature of SBMA, although this finding was discordant when compared with a previous study from this group. ${ }^{4} 14$

'Reverse split-hand syndrome', which refers to the predominant wasting of $\mathrm{ADM}$ and the relative sparing of $\mathrm{APB}$, may suggest the diagnosis of Hirayama disease (HD) in the appropriate clinical context (box 1). HD is described as a distal asymmetrical upper limb amyotrophy with sparing the brachioradialis, thus manifesting as an oblique amyotrophy of forearm muscles. ${ }^{15}$ The specificity of this pattern of amyotrophy to HD is contentious and may lead to misdiagnoses where the prevalence of HD is low.

At a neurophysiological level, an APB/ADM ratio > 1.1, ADM/ $\mathrm{APB}$ ratio $<0.6$, supports the diagnosis of HD. The SI appears less useful for the diagnosis of HD due to a poor specificity having a poor specific when it exceeds 5.2 (34\%). ${ }^{615} 16$ Additionally, the reverse split-hand has also been reported with cervical spondylotic amyotrophy and SMA. ${ }^{4} 17$

The 'split-hand plus' sign has been described and refers to the relative preservation of the flexor pollicis longus muscle, when compared with the thenar muscles (specifically the APB). ${ }^{18} \mathrm{~A}$ split-finger pattern of atrophy has also been described in ALS, whereby the flexor digitorum profundus (FDP) muscle of the index finger exhibit preferential weakness when compared with FDP muscle of the fourth digit (FDP-4). This pattern of weakness is consistent with the split-hand phenomenon, with prominent weakness and amyotrophy of the lateral group of hand muscles. In a clinical setting, the presence of split finger pattern of atrophy may help differentiate ALS from inclusion body myositis where with the latter exhibiting a greater degree of FDP-4 muscle weakness. ${ }^{19}$

In the presence of UMN involvement, patients may develop a pyramidal pattern of weakness, which affects the flexor muscles in the upper limbs ${ }^{20}$ Since biceps brachii has a greater cortical representation than triceps, it may lead to preferential flexor movement weakness: the 'split-elbow'. Although the literature appraising this particular phenomenon is scarce, recent studies emphasised that in upper limbs, the biceps brachii is invariably weaker than of the triceps, irrespective of handedness. ${ }^{21-23}$ More recently, the presence of the split elbow phenomenon was confirmed in a larger study, although contradictory findings have been reported. ${ }^{2324}$

\section{SPLIT SYNDROMES IN THE LOWER LIMBS}

Similar to observations in the hands, lower limb split syndromes have been extensively investigated. While some authors emphasise the predominant amyotrophy of the anterior leg compared with the posterior compartment muscles ${ }^{2526}$ others have reported the opposite. ${ }^{27} 28$ A recent study described a single patient with predominant anterior and posterior muscular compartment leg weakness on different sides, underscoring phenotypic variability. ${ }^{29}$ Notwithstanding the different clinical observations, most authors agree that unlike the 'split-hand syndrome', 'split leg' is primarily a neurophysiological phenomenon. ${ }^{25-29}$

\section{PATHOPHYSIOLOGY}

Four main theories have been put forward to explain the 'splithand' phenomenon, the most studied and best characterised phenomenon of the split phenotypes (figure 1).

\section{Cortical dysfunction}

The notion of cortical basis of the split-hand was suggested by transcranial magnetic stimulation (TMS) studies. Weber and colleagues suggested that preferential dysfunction of corticomotoneuronal inputs to the thenar muscle complex was evident in ALS leading to the split-hand phenomenon. ${ }^{30}$ Additionally, a greater degree of cortical hyperexcitability was documented in the thenar complex muscles (APB/FDI), when compared with $\mathrm{ADM}$, lending further support for the importance of cortical dysfunction in development of the split hand phenomenon in ALS. $^{3}$

Similarly, cortical hyperexcitability was invoked as a likely mechanism for the split-hand plus sign in ALS. ${ }^{31}$ While it could be argued that cortical hyperexcitability may represent a compensatory mechanism in ALS, the finding of a normal cortical excitability in ALS mimicking disorders would argue against such a notion. $^{32}$

\section{Lower motor neuron dysfunction}

Electrophysiology studies appraising CMAP amplitudes and using motor unit number estimation have detected preferential motor unit loss in FDI and APB compared with ADM, which could suggest a specific pattern of LMN susceptibility. ${ }^{4} 73$ Interestingly, the peripheral silent period, obtained by stimulating a digital sensory nerve, was similar in ADM and FDI, not supporting a greater involvement of the LMN innervating FDI muscle. $^{34}$

Preferential APB and FDI atrophy was also confirmed using ultrasound for measuring muscle thickness and calculating an index, and this approach had good accuracy for distinguishing patients with ALS from healthy controls, but not from patients with spinal muscle atrophy. ${ }^{35}$ Similar results were achieved assessing muscle-echo intensity, which could be even more sensitive than the standard electrophysiological index. ${ }^{36}$

Lower motor neuron hyperexcitability

Axonal excitability studies disclosed a preferential increase in the strength duration time constant (SDTC), a biomarker of 
persistent $\mathrm{Na}+$ conductances, in motor axons innervating the APB muscle in patients with ALS. ${ }^{37} 38$

The finding suggested that upregulation of axonal persistent $\mathrm{Na}+$ conductance contributed to development of the split-hand phenomenon, thereby implying a peripheral mechanism. ${ }^{38}$

A subsequent study, assessing the entire neuraxis and recording motor responses from the three muscle groups involved in the split-hand phenomena, reported a comparable increase in axonal excitability that did not follow a split-hand pattern. ${ }^{39} 40$ Taken together, the latter studies suggested that axonal dysfunction was as a downstream process secondary to the intrinsic pathophysiological origins of ALS.

In a recent Japanese study, the split-hand phenomenon was reported in a high proportion of patients with SBMA (57\%), being comparable to ALS (62\%) and higher than disease controls $(20 \%){ }^{13}$

Axonal excitability studies disclosed features of peripheral nerve hyperexcitability in both patients with SBMA and patients with ALS, with SDTC being greater in the former disease. The increase in SDTC was more prominent in patients with SBMA exhibiting a split-hand sign, while no such correlations were evident in ALS. These findings suggested that nerve hyperexcitability was associated with development of the split-hand sign in SBMA, but not ALS. A potential limitation of the Japanese study was that axonal excitability was only assessed from the median nerve, and consequently a split-hand pattern of axonal excitability could not be established. While variable mechanisms could underlie the development of the split-hand phenomenon across different diseases, with axonal hyperexcitability the predominant mechanism in SBMA and cortical hyperexcitability in ALS, future studies assessing excitability from the entire neuraxis and encompassing the affected muscles (APB/ FDI/ADM) would be required.

\section{Motor-end plate dysfunction}

The assessment of clinically unaffected (strong) hands of patients with ALS with normal CMAP amplitudes revealed that the percent of area decrement on repetitive nerve stimulation was significantly greater in APB and FDI compared with healthy controls, but not in ADM. Interestingly, due to physiological reasons, even in control subjects the percent decrement was greater in APB and FDI. ${ }^{41}$ These findings indicate that physiological variability at the neuromuscular junction in small hand muscles may determine differential muscle vulnerability in ALS, potentially contributing to development of the split-hand phenomenon in other neuromuscular disorders.

\section{The split-hand phenomenon: diagnostic and pathogenic relevance in ALS}

Split-hand sign is principally a semiological finding observed in ALS, as described by Wilbourn. ${ }^{2}$ The landmark description of this phenomenon has generated considerable interest because of its potential clinical significance. The quantitative assessment of affected and less affected hand muscles, and the establishment of cut-off values, may be utilised in the diagnostic process. This approach has been refined by incorporation of F-waves persistence, methods for motor unit number estimation and ultrasound. As a diagnostic tool, the relevance of the SI remains a supportive one, especially considering the novel Gold Coast diagnostic criteria which have a high diagnostic utility. ${ }^{42} 43$

As a diagnostic test, the SI may be limited in the setting of severe intrinsic hand muscle atrophy or entrapment neuropathies. Moreover, technical considerations such as optimal electrode placement for measuring CMAP amplitude and temperature control may impact on intra- and inter-rater reliability. ${ }^{44}$ Multicenter studies would be required to assess the utility of the SI in a clinical setting.

More importantly, split-hand phenomenon may offer pathophysiological insights into ALS. Despite being observed in ALS mimicking disorders, motor cortical dysfunction is likely to significantly contribute to development of the split-hand phenomenon. ${ }^{1114}$ The interest in the underlying mechanisms has driven motivation to detect other split-limb phenomena in ALS, although most of these are not readily observed in clinical practice. Increasingly, it is accepted that a long preclinical period of neuronal dysfunction precedes overt weakness and that considerable adaptive processes take place in corticomotoneuronal circuits. ${ }^{45}$

Despite advances in ALS research, basic pathophysiology questions remain open and subject of intense debate; are UMN and LMN degeneration interlinked or do they evolve independently from one another? If there is a biological association between UMN and LMN degeneration, does this represent a 'dying back' or a 'dying forward' process? The dying forward hypothesis could potentially explain that occurrence of the split-hand sign in ALS. The lateral hand groups of muscles (APB/FDI) exhibit a greater anatomical and function cortical representation, in part related to evolution of specialised activity of these muscles. ${ }^{46-50}$ Consequently, the corticomotoneuronal projections could lead to a greater degree of cortical hyperexcitability and thereby predisposition for neurodegeneration of the spinal motor neurons innervating the lateral hand groups of muscles via an anterograde glutamatergic mechanism. ${ }^{46}$ Support for a dying forward hypothesis mechanism was provided by clinical observation of relative sparing of the oculomotor and Onuf's nuclei in ALS, which do not directly synapse with the corticospinal tract. ${ }^{51}$ Additionally, TMS studies have established cortical hyperexcitability as an early and specific feature of ALS, correlating with patterns of disease spread and the split-hand sign. ${ }^{52-56}$ Importantly, neuropathology studies have also provided additional evidence by established the presence of TDP-43 aggregation in $\sim 97 \%$ of all ALS cases. ${ }^{57}$ The TDP-43 inclusions are evident in demented and non-demented patients with ALS and increase in density with disease evolution, particularly development of cognitive impairment. ${ }^{58}$ Consequently, a cortical basis for the split-hand phenomenon could explain the typical topography of hand muscle wasting in ALS, which cannot be explained by radicular, plexus and nerve trunk territories.

It should be acknowledged that our interpretation could be influenced by a simple descending pathway model. Given that the split-hand phenomenon is not a universal finding in patients with ALS and may be observed in neurological disorders without UMN involvement (such as SBMA), the possibility of a combined contribution of UMN and LMN dysfunction needs to be considered and that their influence may change during the disease progression. Future studies should combine a large set of cortical, spinal cord, axonal and end-plate investigations to dissect the factors involved in its onset and progression.

Correction notice This article has been corrected since it appeared Online First. Author affiliations for Peter Bede have been corrected.

Acknowledgements We thank "Cooperative Artefacts" who designed the picture Contributors All authors contributed equally to the inception and drafting of this review paper.

Funding The authors have not declared a specific grant for this research from any funding agency in the public, commercial or not-for-profit sectors. 
Competing interests None declared.

Patient consent for publication Not required.

Provenance and peer review Commissioned; externally peer reviewed.

ORCID iDs

Philippe Corcia http://orcid.org/0000-0002-1625-8845

Peter Bede http://orcid.org/0000-0003-0492-4772

Steve Vucic http://orcid.org/0000-0002-8323-873X

\section{REFERENCES}

1 Hardiman 0, Al-Chalabi A, Chio A. Amyotrophic lateral sclerosis. Nat Rev Dis Primer 2017:3:17085.

2 Wilbourn AJ. The "split hand syndrome". Muscle Nerve 2000;23:138.

3 Menon P, Kiernan MC, Yiannikas C, et al. Split-Hand index for the diagnosis of amyotrophic lateral sclerosis. Clin Neurophysio/ 2013:124:410-6.

4 Kuwabara S, Sonoo M, Komori T, et al. Dissociated small hand muscle atrophy in amyotrophic lateral sclerosis: frequency, extent, and specificity. Muscle Nerve 2008;37:426-30.

5 Fang J, Liu M-S, Guan Y-Z, et al. Pattern differences of small hand muscle atrophy in amyotrophic lateral sclerosis and mimic disorders. Chin Med J 2016;129:792-8.

6 Kalita J, Kumar S, Misra UK, et al. Split hand index and ulnar to median ratio in Hirayama disease and amyotrophic lateral sclerosis. Amyotroph Lateral Scler Frontotemporal Degener 2017;18:598-603.

7 Wang Z-L, Liu M, Cai Z, et al. A prospective study on split-hand index as a biomarker for the diagnosis of amyotrophic lateral sclerosis. Amyotroph Lateral Scler Frontotemporal Degener 2020;21:574-83.

8 Wang Z-L, Cui L, Liu M, et al. Split-Hand syndrome in amyotrophic lateral sclerosis: differences in dysfunction of the Fdl and ADM spinal motoneurons. Front Neurosci 2019;13:371.

9 Nandedkar SD, Nandedkar DS, Barkhaus PE, et al. Motor unit number index (MUNIX). IEEE Trans Biomed Eng 2004;51:2209-11.

10 Kim D-G, Hong Y-H, Shin J-Y, et al. Split-Hand phenomenon in amyotrophic lateral sclerosis: a motor unit number index study. Muscle Nerve 2016;53:885-8.

11 Gourie-Devi M, Suresh TG, Shankar SK. Monomelic amyotrophy. Arch Neurol 1984:41:388-94.

12 Kang YR, Kang KW, Nam TS, et al. Split Hand Syndrome in a Family with GARS Associated Axonal Neuropathy. J Clin Neurol 2019:15:566-8.

13 Schelhaas HJ, van de Warrenburg BPC, Kremer HPH, et al. The "split hand" phenomenon: evidence of a spinal origin. Neurology 2003:61:1619-20.

14 Shibuya K, Misawa S, Uzawa A, et al. Split hand and motor axonal hyperexcitability in spinal and bulbar muscular atrophy. J Neurol Neurosurg Psychiatry 2020;91:1189-94.

15 Singh R-J, Preethish-Kumar V, Polavarapu K, et al. Reverse split hand syndrome: dissociated intrinsic hand muscle atrophy pattern in Hirayama disease/brachial monomelic amyotrophy. Amyotroph Lateral Scler Frontotemporal Degener 2017; 18:10-16

16 Jin $X$, Jiang J-Y, Lu F-Z, et al. Electrophysiological differences between Hirayama disease, amyotrophic lateral sclerosis and cervical spondylotic amyotrophy. BMC Musculoskelet Disord 2014:15:349.

17 Günther R, Neuwirth C, Koch JC, et al. Motor unit number index (MUNIX) of hand muscles is a disease biomarker for adult spinal muscular atrophy. Clin Neurophysiol 2019;130:315-9.

18 Menon P, Bae JS, Mioshi E, et al. Split-Hand plus sign in ALS: differential involvement of the flexor pollicis longus and intrinsic hand muscles. Amyotroph Latera/ Scler Frontotemporal Degener 2013;14:315-8.

19 Sonoo M, Takahashi K, Hamada Y, et al. Split-finger syndrome in amyotrophic lateral sclerosis. J Neurol Neurosurg Psychiatry 2020;91:1235-6.

20 Wiles CM. Pyramidal weakness. Pract Neurol 2017:17:241-2.

21 Khalaf R, Martin S, Ellis C, et al. Relative preservation of triceps over biceps strength in upper limb-onset ALS: the 'split elbow'. J Neurol Neurosurg Psychiatry 2019;90:730-3

22 Vucic S. Split elbow sign: more evidence for the importance of cortical dysfunction in ALS. J Neurol Neurosurg Psychiatry 2019;90:729.

23 Ludolph AC, Emilian S, Dreyhaupt J, et al. Pattern of paresis in ALS is consistent with the physiology of the corticomotoneuronal projections to different muscle groups. $J$ Neurol Neurosurg Psychiatry 2020;91:991-8.

24 Liu J, Wang Z, Shen D, et al. Split phenomenon of antagonistic muscle groups in amyotrophic lateral sclerosis: relative preservation of flexor muscles. Neurol Res 2021:43:372-80.

$25 \mathrm{Hu}$ F, Jin J, Chen Q, et al. Dissociated lower limb muscle involvement in amyotrophic lateral sclerosis and its differential diagnosis value. Sci Rep 2019:9:17786.

26 Wang Z-L, Cui L, Liu M, et al. Reassessment of split-leg signs in amyotrophic latera sclerosis: differential involvement of the extensor digitorum brevis and abductor hallucis muscles. Front Neurol 2019;10:565.
27 Simon NG, Lee M, Bae JS, et al. Dissociated lower limb muscle involvement in amyotrophic lateral sclerosis. J Neurol 2015;262:1424-32.

28 Min YG, Choi S-J, Hong Y-H, et al. Dissociated leg muscle atrophy in amyotrophic lateral sclerosis/motor neuron disease: the 'split-leg' sign. Sci Rep 2020;10:15661.

29 de Carvalho M, Swash M. The "split-leg" syndrome in ALS: specific or variable? Amyotroph Lateral Scler Frontotemporal Degener 2019:20:615-6.

30 Weber M, Eisen A, Stewart H, et al. The split hand in ALS has a cortical basis. J Neurol Sci 2000;180:66-70.

31 Bae JS, Menon P, Mioshi E, et al. Cortical hyperexcitability and the split-hand plus phenomenon: pathophysiological insights in ALS. Amyotroph Lateral Scler Frontotemporal Degener 2014;15:250-6.

32 Menon P, Geevasinga N, Yiannikas C, et al. Sensitivity and specificity of threshold tracking transcranial magnetic stimulation for diagnosis of amyotrophic lateral sclerosis: a prospective study. The Lancet Neurology 2015;14:478-84.

33 Kuwabara S, Mizobuchi K, Ogawara K, et al. Dissociated small hand muscle involvement in amyotrophic lateral sclerosis detected by motor unit number estimates. Muscle Nerve 1999:22:870-3.

34 Cengiz B, Mercan M, Kuruoğlu R. Spinal excitability changes do not influence the mechanisms of split-hand syndrome in amyotrophic lateral sclerosis. Muscle Nerve 2018;58:503-8.

35 Abraham A, Fainmesser Y, Drory VE, et al. Split-Hand phenomenon in motor neuron diseases: sonographic assesment of muscle thickness. Clin Neurophysiol 2020;131:1721-5.

36 Seok HY, Park J, Kim YH, et al. Split hand muscle echo intensity index as a reliable imaging marker for differential diagnosis of amyotrophic lateral sclerosis. J Neurol Neurosurg Psychiatry 2018;89:943-8.

37 Kiernan MC, Bostock H, Park SB, et al. Measurement of axonal excitability: consensus guidelines. Clin Neurophysiol 2020;131:308-23.

38 Shibuya K, Misawa S, Nasu S, et al. Split hand syndrome in amyotrophic lateral sclerosis: different excitability changes in the thenar and hypothenar motor axons. J Neurol Neurosurg Psychiatry 2013;84:969-72.

39 Menon P, Kiernan MC, Vucic S. Cortical dysfunction underlies the development of the split-hand in amyotrophic lateral sclerosis. PLoS One 2014a;9:e87124.

40 Menon P, Kiernan MC, Vucic S. Als pathophysiology: insights from the split-hand phenomenon. Clin Neurophysiol 2014;125:186-93.

41 de Carvalho M, Swash M. The split hand in amyotrophic lateral sclerosis: a possible role for the neuromuscular junction. Amyotroph Lateral Scler Frontotemporal Degener 2019;20:368-75

42 Shefner JM, Al-Chalabi A, Baker MR, et al. A proposal for new diagnostic criteria for ALS. Clin Neurophysiol 2020;131:1975-8.

43 Hannaford A, Pavey N, van den Bos M. Diagnostic utility of gold Coast criteria in ALS. Ann Neurol 2021;89:979-86.

44 Bromberg MB, Spiegelberg T. The influence of active electrode placement on CMAP amplitude. Electroencephalogr Clin Neurophysiol 1997;105:385-9.

45 Eisen A, Kiernan M, Mitsumoto H, et al. Amyotrophic lateral sclerosis: a long preclinical period? J Neurol Neurosurg Psychiatry 2014;85:1232-8.

46 Eisen $\mathrm{A}$. The dying forward hypothesis of ALS: tracing its history. Brain SC 2021;11:300.

47 Abbruzzese G, Assini A, Buccolieri A, et al. Comparison of intracortical inhibition and facilitation in distal and proximal arm muscles in humans. J Physiol 1999;514 (Pt 3):895-903

48 Lemon RN, Griffiths J. Comparing the function of the corticospinal system in different species: organizational differences for motor specialization? Muscle Nerve 2005:32:261-79.

49 Lemon RN. What drives corticospinal output? F1000 Biol Rep 2010;2:51.

50 Menon P, Kiernan MC, Vucic S. Cortical excitability differences in hand muscles follow a split-hand pattern in healthy controls. Muscle Nerve 2014;49:836-44.

51 Hudson AJ, Kiernan JN. Preservation of certain voluntary muscles in motoneurone disease. Lancet 1988;1:652-3.

52 Vucic S, Kiernan MC. Novel threshold tracking techniques suggest that cortical hyperexcitability is an early feature of motor neuron disease. Brain 2006; 129:2436-46

53 Vucic S, Cheah BC, Yiannikas C, et al. Corticomotoneuronal function and hyperexcitability in acquired neuromyotonia. Brain 2010;133:2727-33.

54 Vucic S, Nicholson GA, Kiernan MC. Cortical hyperexcitability may precede the onset of familial amyotrophic lateral sclerosis. Brain 2008;131:1540-50.

55 Menon P, Geevasinga N, van den Bos M, et al. Cortical hyperexcitability and disease spread in amyotrophic lateral sclerosis. Eur J Neurol 2017;24:816-24.

56 Menon $\mathrm{P}$, Higashihara M, van den Bos M, et al. Cortical hyperexcitability evolves with disease progression in ALS. Ann Clin Trans/ Neurol 2020;7:733-41.

57 Tan RH, Ke YD, Ittner LM, et al. Als/Ftld: experimental models and reality. Acta Neuropathol 2017;133:177-96.

58 Wilson CM, Grace GM, Munoz DG, et al. Cognitive impairment in sporadic ALS: a pathologic continuum underlying a multisystem disorder. Neurology 2001;57:651-7. 